\title{
Influence of Curvature on Air-Gap Magnetic Field Distribution and Rotor Losses in PM Electric Machines
}

\author{
J. R. Anglada *, S. M. Sharkh *, and A. A. Qazalbash** \\ *Mechatronics Research Group, Engineering Sciences, University of Southampton, Southampton, \\ U.K., email: \{j.renedo-anglada,suleiman\}@soton.ac.uk \\ ** Emerson Control Techniques Dynamics Ltd, Andover, Hampshire, U.K., \\ email: arfakhshand.qazalbash@hotmail.com
}

December 12, 2016

\begin{abstract}
Purpose - The purpose of this paper is to study the effect of curvature on the magnetic field distribution and no-load rotor eddy-current losses in electric machines, particularly in the case of high-speed permanent magnet machines.

Design/methodology/approach - The magnetic field distribution is obtained using conformal mapping and eddycurrent losses are obtained using a cylindrical multilayer model. The analytical results are validated using 2D FEA. The analytical method is based on a proportional-logarithmic conformal transformation that maps the cylindrical geometry of a rotating electric machine into a rectangular configuration without modifying the length scale. In addition, the appropriate transformation of permanent magnet (PM) cylindrical domains into the rectangular domain is deduced. Based on this conformal transformation a coefficient to quantify the effect of curvature is proposed.

Findings - Neglecting the effect of curvature can produce significant errors in the calculation of no-load rotor losses when the ratio between the air-gap length and the diameter of the rotor is large.

Originality/value - The appropriate transformation of permanent magnet (PM) cylindrical domains into the rectangular domain is deduced. The proportional-logarithmic transformation proposed provides an insight into the effect of curvature on the magnetic field distribution in the air-gap and no-load rotor losses. Furthermore, the proposed curvature coefficient gives a notion of the effect of curvature for any particular geometry without the necessity of any complicated calculation. The case study shows that neglecting the effect of curvature underestimates the rotor eddycurrent losses significantly in machines with large gap to rotor diameter ratios.
\end{abstract}

Keywords Conformal mapping, permanent magnet machines, rotor eddy-current power loss calculation.

Paper type Research paper

\section{Introduction}

The traditional approach for studying the magnetic field in electric machines has been simply to cut and open the rotor and the stator to produce a rectangular developed model neglecting curvature (Kundur, 1993; Fitzgerald et al., 2003; Matsch, 1972). The results are acceptable for devices with great radii and small gaps. However, in small machines with relatively large air-gaps, like permanent-magnet (PM) machines with retaining sleeves, this approximation is not sufficiently accurate. Several parameters of the machine like torque ripple and rotor losses (Sharkh et al., 2011; Qazalbash et al., 2014a,b; Zhu et al., 2004) are strongly affected by the harmonics of the air-gap magnetic field. In cases like this, the results of the magnetic field calculations should be as precise as possible for accurate performance prediction.

High accuracy of rotor loss calculation is very important as they are usually very small compared to the rating of a machine. For example, a commercial $60 \mathrm{~kW}, 60 \mathrm{krpm}$ machine would not be feasible if the losses are more than about 
$100 \mathrm{~W}$. Underestimating the losses would result in a design that may fail in practice. Overestimating them may result in ruling out a feasible and maybe good machine design.

As will be seen later in this paper, the effect of curvature on rotor losses can be very significant, especially in machines with large diameter to air-gap ratios; the curvature tends to increase losses by as much as $100 \%$ in some case compare to those estimated using a developed rectilinear model. However, while there are many papers such as (Rabinovici, 1996), which deals with the effect of curvature on air-gap flux distribution, a clear criterion as to when is it reasonable to ignore the effect of curvature or its effect on rotor losses have not been addressed.

Numerical methods, like finite element analysis (FEA), can of course be used to produce accurate solutions using the real geometry. However, they do not readily provide an insight into the effect of machine parameters on field quantities and performance. For this reason, analytical methods remain very useful as a first approach in the design optimisation process.

The background theory of the analytical method presented in this paper is conformal mapping. The idea is to use complex functions to transform a complicated domain into a simpler one in which the solution is known (Gibbs, 1958; Freeman, 1962; Zhu and Howe, 1993). To account for slotting, conformal mapping can be used to obtain a complex permeance (CP) function that modulates the magnetic field of a slotless solution (Zarko et al., 2006; Boughrara et al., 2009). An alternative method for obtaining the magnetic field distribution in the air-gap is the sub-domain method (Dubas and Espanet, 2009; Wu et al., 2010; Zhu et al., 2010; Wu et al., 2011, 2012), which provides an accurate solution but less insight because of the complicated mathematics involved.

As mentioned earlier, the problem of modelling the effect of curvature analytically was addressed by Rabinovici (Rabinovici, 1996) using a pure logarithmic transformation that maps the circular geometry into a rectangular one, which can be solved using conformal mapping techniques like those developed by Gibbs (Gibbs, 1958) and Freeman (Freeman, 1962) based on the Schwarz-Christoffel transformation. However, a pure logarithmic transformation makes the length of the new geometry completely different from the real one thus making it difficult to assess how strong the effect of curvature is or what would happen if it is ignored. Similar methods were also used in (Zarko et al., 2006; Boughrara et al., 2009; Markovic et al., 2004); although these produce a solution to the problem, they do not provide a direct insight into the effect of curvature partly due to the change in the length scale.

In this paper, a proportional-logarithmic conformal transformation is proposed to transform the cylindrical geometry into a rectangular one with the same length scale as the real geometry and the traditional developed rectilinear model. The paper also derives the appropriate correct transformation of permanent magnet (PM) in the cylindrical domain into the rectangular one, which has not been done before. The new rectangular developed model can be solved using the usual methods. Because of the dimensional similarity of the new rectangular model and the actual machine, the effect of curvature can be easily understood.

Carter (Carter, 1900, 1926) proposed a coefficient that shows how the effect of slotting can be modelled as an increase of the air-gap length. This paper proposes a similar coefficient that can be applied to quantify the effect of curvature. The coefficient is defined as the ratio of the air-gap lengths of the equivalent rectilinear model over the air-gap length of the actual cylindrical geometry. This coefficient can be used to assess beforehand if the effect of curvature is going to be significant and accordingly choose the appropriate model for the analysis.

The method is applied to the calculation of asynchronous harmonics and corresponding rotor losses in two examplar high speed PM machines: one with a large gap to diameter ratio and hence a strong curvature effect and the other has double the diameter and the same air-gap and a less pronounced effect of curvature.

The paper makes the following novel contributions:

- It introduces a modified conformal transformation of cylindrical geometries into rectilinear ones, which preserve their length scale.

- A curvature coefficient, analogous to Carter's coefficient, is defined to enable a simple assessment of the effect of curvature.

- The paper also presents a corrected transformation of cylindrical PM domains into the rectangular domain.

- This methodology is applied to the analysis of the magnetic field in two PM machines to calculate the asynchronous harmonics and associated rotor losses. The results show that the effect of curvature can be very 
significant.

\section{Effect of Curvature on the Magnetic Field}

Fig. 1 shows a generic cross-section of a rotating electric machine with a toothed stator and a smooth interior rotor. One tooth-pitch, $\tau_{z}=s_{z}+t_{z}$. In Fig. $1 s_{z}$ is the slot pitch, $t_{z}$ the tooth pitch, $g_{z}$ the effective air-gap length, $R_{r}$ the rotor radius and $R_{s}$ the stator bore radius. The effective air-gap length is defined as the magnet thickness plus the clearance gap plus the sleeve thickness.

This generic geometry ignores the tooth tips; the top of the slot is assumed to be wide as a the slot opening and the sides of the slots are assumed to be radial. These are reasonable assumptions if the teeth, particularly the teeth tips, are not saturated, which is usually the case in machines of good design.

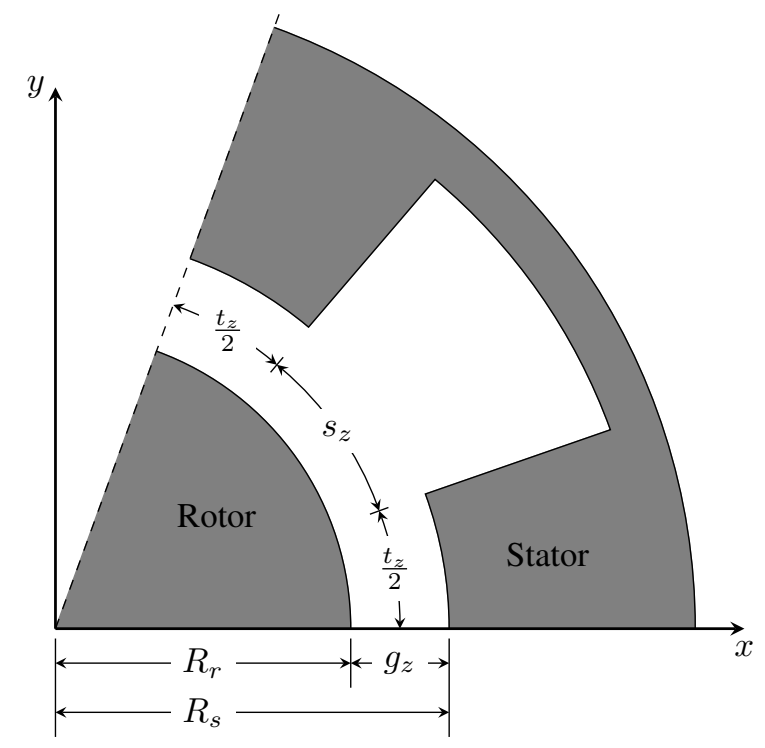

Figure 1: Cylindrical geometry studied showing only one slot in the $z$-plane.

The geometry in the $z$-plane in Fig. 1 can be transformed into that shown in the $w$-plane in Fig. 2 using a logarithmic transformation, as proposed by Rabinovici (Rabinovici, 1996):

$$
w=R \ln (z)=R \ln \left(r_{z}\right)+j R \theta_{z},
$$

where $R$ is a proportionality constant, $z=r_{z} e^{j \theta_{z}}$ is the complex variable in the $z$-plane and $w$ the complex variable in the $w$-plane.

Rabinovici implicitly sets $R=1$ in the above equation. However, this makes the $w$-plane geometry in Fig. 2 very different in its scale from that in Fig. 1. The vertical length of the geometry in the $w$-plane is equal to the angle of one tooth pitch, i. e. $\tau_{w}=\theta_{\tau}$, where $\theta_{\tau}$ is the tooth pitch angle.

In this paper we propose to set $R$ to be the radius in the middle of the air-gap, $R_{g}=R_{r}+\frac{g_{z}}{2}$. This results in

$$
\begin{gathered}
t_{w}=R_{g} \theta_{t}=t_{z}, \\
s_{w}=R_{g} \theta_{s}=s_{z}, \\
\tau_{w}=R_{g} \theta_{\tau}=\tau_{z},
\end{gathered}
$$




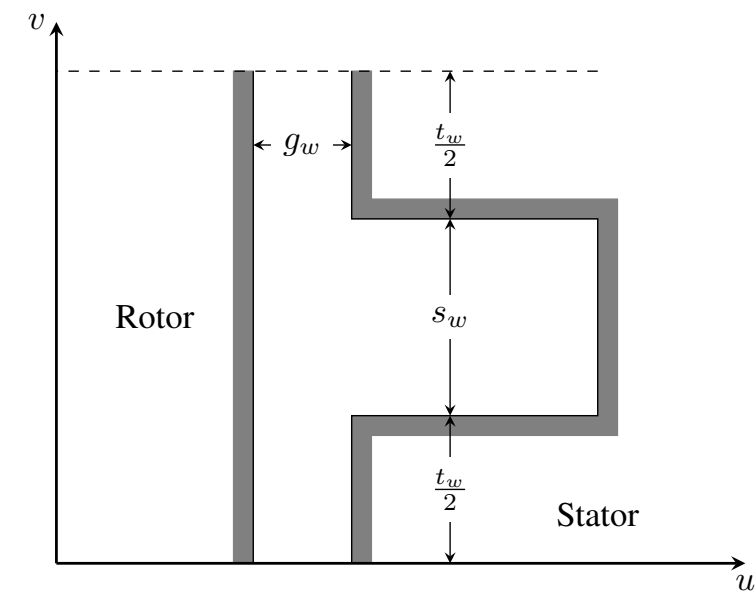

Figure 2: Rectangular developed model obtained after the proposed transformation in the $w$-plane.

$$
g_{w}=R_{g}\left[\ln \left(R_{s}\right)-\ln \left(R_{r}\right)\right]=R_{g} \ln \left(\frac{R_{s}}{R_{r}}\right),
$$

where $\theta_{t}$ and $\theta_{s}$ are the tooth and slot angles, respectively. From these equations it can be easily appreciated that all the geometrical length parameters are exactly the same as in Fig. 1 with the exception of $g_{w}$.

It can be readily shown that $g_{w}$ can be expressed in terms of $g_{z}$ and $R_{g}$ as follows:

$$
g_{w}=R_{g} \ln \left(\frac{R_{g}+\frac{g_{z}}{2}}{R_{g}-\frac{g_{z}}{2}}\right) .
$$

After these first manipulations comes the first intuitive interpretation of this transformation. The geometry obtained from the proposed conformal transformation is exactly the same as the conventional developed model that is obtained simply by cutting and opening the machine. The only difference is in the parameter $g_{w}$ whose value is given by (6).

\subsection{Curvature Coefficient}

Carter (Carter, 1900, 1926) proposed that the effect of slotting can be represented as a modification of the air-gap length of an equivalent slotless model. The effect of slotting on the mean air-gap flux density can be accounted by multiplying the air-gap length, $g$, by Carter's coefficient, $K_{c}$, (Krause et al., 2013) to obtain:

$$
g^{\prime}=g K_{c},
$$

which is set to be the gap of of an equivalent slotless model. The mean value of the magnetic field in the slotted machine is then calculated as

$$
B_{s-\text { slotted }}=\frac{\mu_{0} F}{g^{\prime}} .
$$

where $F$ is the mmf drop across the gap, $g$ of the slotless model. If we define $B_{s-s l o t l e s s}$ as the mean value of the magnetic field of a slotless machine with a gap of $g$, then

$$
B_{s-\text { slotless }}=\frac{\mu_{0} F}{g} .
$$

Hence

$$
B_{s-\text { slotted }}=\frac{B_{s-\text { slotless }}}{K_{c}}
$$


It was deduced previously, from (2)-(6), that using the proposed transformation the rectangular geometry obtained has the same basic dimensions as the original cylindrical geometry except for the air-gap length $g_{w}$. Following the same logic as that used when defining Carter's coefficient we define a curvature coefficient $K_{J}$ such that

$$
g_{w}=g_{z} K_{J} \rightarrow K_{J}=\frac{g_{w}}{g_{z}} .
$$

Applying (11) to the mean value of the magnetic field:

$$
B_{s-c u r v}=\frac{\mu_{0} F}{g_{w}}=\frac{B_{s-r e c t}}{K_{J}} .
$$

Substituting (6) into (11) we obtain the following expression for $K_{J}$ as a function of the ratio between the radius of the machine and the air-gap length, $R_{g} / g_{z}$ :

$$
K_{J}=\frac{R_{g}}{g_{z}} \ln \left(\frac{\frac{R_{g}}{g_{z}}+\frac{1}{2}}{\frac{R_{g}}{g_{z}}-\frac{1}{2}}\right) .
$$

With this function a direct estimation of the influence of curvature on the mean value of the flux density can be obtained without any other calculation; the effect will depend on the ratio of $g_{z}$ and $R_{g}$. Qualitatively this means that if the air-gap length is large compared to the radius of the machine the effect of curvature is significant and when the radius is considerably larger than the air-gap length this effect is negligible.

Like Carter's coefficient, the value of $K_{J}$ is close 1 ; it equals 1 for a rectangular geometry and it becomes slightly grater than one as the curvature increases. The difference between the value of $K_{J}$ and 1, i.e., $K_{J}-1$, would therefore provide a measure of the effect of curvature on the magnetic field. Fig. 3 shows a graph of $\left(K_{J}-1\right)$ in percentage versus the ratio of the radius in the middle of the gap to that air-gap length. As expected, the influence of curvature reduces as the radius increases for a given air-gap length. Below a ratio of about 6 the effect of curvature is expected to be significant.

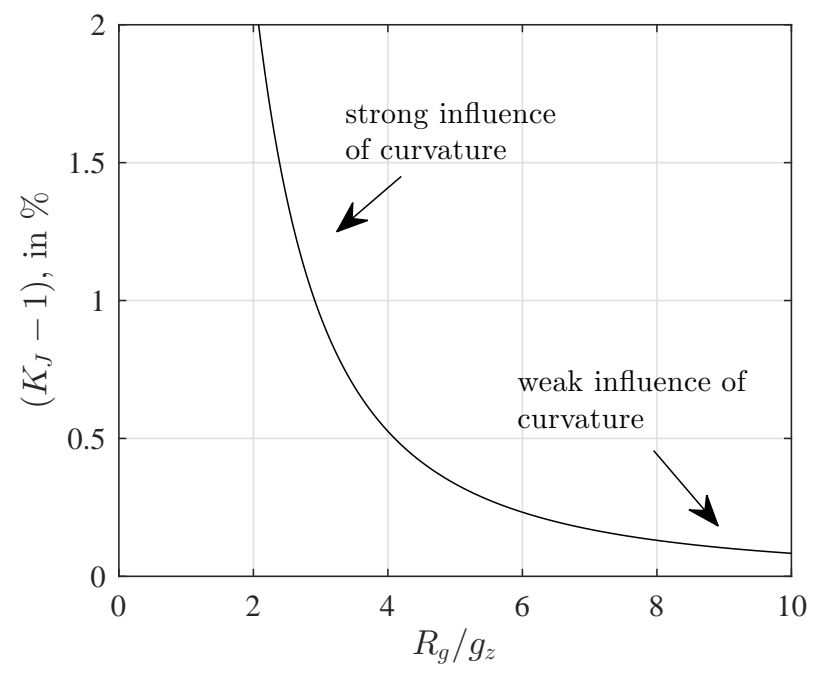

Figure 3: The curvature coefficient, $K_{J}$, as a function of the ratio between the air-gap radius and the air-gap length.

\subsection{Magnetic Field Relations}

Using the conformal transformation we can obtain the relationship between the magnetic field in the $z$-plane and the $w$-plane. With this relationship the solution of the rectilinear geometry in the $w$-plane can be transformed into 
a solution for the real cylindrical geometry in the $z$-plane. To deduce these equations the magnetic scalar potential functions in both planes are considered (Hammond, 1999; Zarko et al., 2006; Boughrara et al., 2009; Markovic et al., 2004, 2005):

- $\varphi(x, y) \equiv$ scalar potential in the $z$-plane.

- $\psi(u, v) \equiv$ scalar potential in the $w$-plane.

Each point $(u, v)$ in $w$ is at the same potential as the corresponding point $(x, y)$ in $z$ and hence the following equation must be satisfied in all the domain:

$$
\varphi(x, y)=\psi(u(x, y), v(x, y)) .
$$

The field intensity in the $z$-plane is obtained from the potential function in the $z$-plane, $\varphi(x, y)$, as follows:

$$
H_{z}=H_{x}+j H_{y}=-\frac{\partial \varphi}{\partial x}-j \frac{\partial \varphi}{\partial y} .
$$

The field intensity in the $w$-plane is obtained from the potential function in the $w$-plane, $\psi(u, v)$, as follows:

$$
H_{w}=H_{u}+j H_{v}=-\frac{\partial \psi}{\partial u}-j \frac{\partial \psi}{\partial v} .
$$

Applying the rule of an implicit derivative to the scalar potential we obtain:

$$
\begin{aligned}
& \frac{\partial \varphi}{\partial x}=\frac{\partial \psi}{\partial u} \frac{\partial u}{\partial x}+\frac{\partial \psi}{\partial v} \frac{\partial v}{\partial x} \\
& \frac{\partial \varphi}{\partial y}=\frac{\partial \psi}{\partial u} \frac{\partial u}{\partial y}+\frac{\partial \psi}{\partial v} \frac{\partial v}{\partial y} .
\end{aligned}
$$

Combining (15), (17) and (18) it can be shown that:

$$
H_{z}=H_{u} \frac{\partial u}{\partial x}+H_{v} \frac{\partial v}{\partial x}+j\left(H_{u} \frac{\partial u}{\partial y}+H_{v} \frac{\partial v}{\partial y}\right) .
$$

As potential functions are analytic they satisfy the Cauchy-Riemann conditions ${ }^{1}$ (Hammond, 1999; Zarko et al., 2006) and hence (19) they can be rewritten as

$$
H_{z}=\left(H_{u}+j H_{v}\right)\left(\frac{\partial u}{\partial x}-j \frac{\partial v}{\partial x}\right),
$$

or

$$
H_{z}=H_{w}\left(\frac{\partial u}{\partial x}-j \frac{\partial v}{\partial x}\right) .
$$

Considering that $w=u(x, y)+j v(x, y)$, then

$$
\frac{\partial w}{\partial x}=\frac{\partial u}{\partial x}+j \frac{\partial v}{\partial x}=\frac{\partial w}{\partial z} \frac{\partial z}{\partial x}=\frac{d w}{d z} .
$$

\footnotetext{
${ }^{1}$ The Cauchy-Riemann conditions are:

$$
\begin{aligned}
& \frac{\partial u}{\partial x}=\frac{\partial v}{\partial y} \\
& \frac{\partial u}{\partial y}=\frac{\partial v}{\partial x}
\end{aligned}
$$
}


The complex conjugate

$$
\left(\frac{d w}{d z}\right)^{*}=\left(\frac{\partial u}{\partial x}+j \frac{\partial v}{\partial x}\right)^{*}=\frac{\partial u}{\partial x}-j \frac{\partial v}{\partial x}
$$

If (23) is substituted in (21), the relationship of the field intensity in the $w$-plane with the $z$-plane is then given by

$$
H_{z}=H_{w}\left(\frac{d w}{d z}\right)^{*}
$$

Since $B=\mu_{0} H$ in the air-gap, then

$$
B_{z}=B_{w}\left(\frac{d w}{d z}\right)^{*}
$$

From (1):

$$
\frac{d w}{d z}=\frac{R_{g}}{z}=\frac{R_{g}}{r_{z}} e^{-j \theta_{z}}
$$

Substituting (26) in (25) yields

$$
B_{z}=B_{w}\left\{\frac{R_{g}}{r_{z}} e^{j \theta_{z}}\right\}=B_{w}\left\{M\left(r_{z}\right) e^{j \theta_{z}}\right\} .
$$

The second term of (27), $e^{-j \theta_{z}}$, simply rotates and aligns the radial direction in the $z$-plane with the horizontal axis in the $w$-plane. It does not affect the value of the field and therefore it will not be analysed further.

The term $M=R_{g} / r_{z}$ can be interpreted as a scale factor, related with the flux focusing effect of curvature. Because of the nature of the transformation the region $r_{z}<R_{g}$ is contracted, which intensifies the field. In the region $r_{z}>R_{g}$, which is expanded compared to the rectangular model, the field's intensity is reduced. When $r_{z}=R_{g}$ the magnitude of the magnetic field is not altered.

The value of the scale factor in the $z$-plane therefore defines the influence of curvature on the magnitude of the magnetic field density at a particular point. Fig. 4 shows a 2D representation of $M$ in a certain region of the $z$-plane. The value of $M\left(r_{z}\right)$ was restricted to values between 0.5 and 1.5 because usually the value of $M$ in the air-gap (the region of interest in this case) is within these limits.

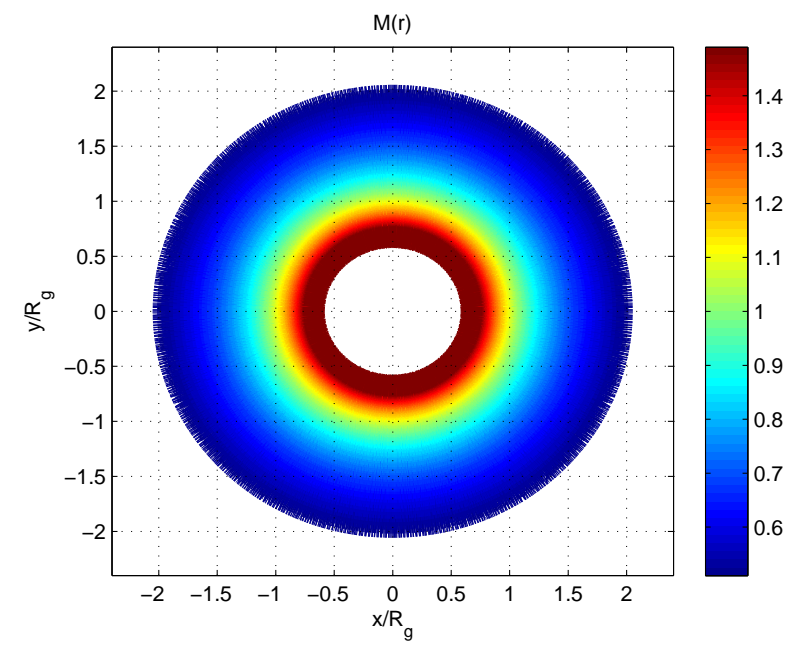

Figure 4: $M\left(r_{z}\right)=\frac{R_{g}}{r_{z}}$. 
This representation of the scale factor $M\left(r_{z}\right)$ shows how the magnetic field is intensified in some regions and debilitated in others. As the radius reduces, the field lines are bunched closer together, thus intensifying the magnetic field, and vice versa. If the air-gap length is small and it is contained within the light green region the effect of curvature will be small. However, if this is not the case the effect of the scale factor will be important - neglecting the curvature gives inaccurate results. The proportionality constant $R_{g}$ will determine the radius at which the scale factor is unity, the radius where distances are not distorted.

\section{Permanent Magnet Transformation}

In this section the transformation equations for a radially magnetised permanent magnet are deduced based on the assumption that these permanent magnets can be represented by two current sheets on the edges of each magnet (Boules, 1985; Rabinovici, 1996), with a current density $J(\mathrm{~A} / \mathrm{m})$ equal to the magnetisation of the material, $J=\mathcal{M}$. A permanent magnet in the $z$-plane is represented in Fig. 5 with the equivalent current sheets as dots and crosses.

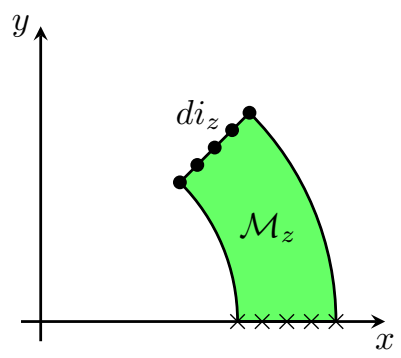

Figure 5: Permanent magnet in the cylindrical geometry, the $z$-plane.

Rabinovici (Rabinovici, 1996) proposes that a cylindrical permanent magnet in the $z$-plane is transformed into a rectangular one in the $w$-plane such that the magnetisation $\mathcal{M}_{w}$ in the $w$-plane is set in such a way that the total equivalent current is the same in both planes. The new permanent magnet in the $w$-plane is shown in Fig. 6 . The implicit assumption in this procedure is that the current density is constant along the edge of the magnets in the $w$-plane, i.e., that $\mathcal{M}_{w}$ is constant. It is shown below that this is not correct.

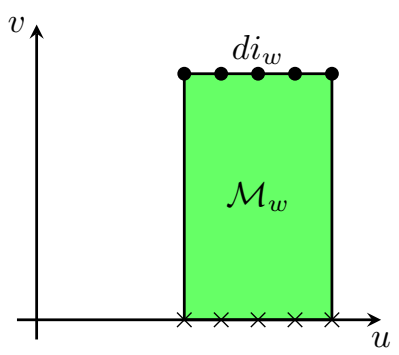

Figure 6: Permanent magnet in the rectangular developed geometry, the $w$-plane.

For the deduction of the transformation equations let us consider the permanent magnet in the $z$-plane shown in Fig. 5 and the transformed magnet in the $w$-plane, Fig. 6. The equivalent current sheets of the magnet in the $z$-plane have a constant current density $J_{z}$. To represent these two current sheets in the $w$-plane each differential current point, $d i_{z}$, is mapped on the $w$-plane in the corresponding position (Rabinovici, 1996; Boughrara et al., 2009). The magnitude of the current should be same in both planes, this is

$$
d i_{z}=d i_{w}
$$

Each of these differential currents can be expressed in terms of the corresponding current density

$$
d i_{z}=J_{z} d r_{z}
$$




$$
d i_{w}=J_{w} d u
$$

Combining (28), (29) and (30) yields

$$
J_{z} d r_{z}=J_{w} d u \Rightarrow J_{w}=J_{z}\left(\frac{d u}{d r_{z}}\right) .
$$

The derivative can be obtained from (1). The final expression of the new current density is the following:

$$
J_{w}\left(r_{z}\right)=J_{z}\left(\frac{r_{z}}{R_{g}}\right)=\frac{J_{z}}{M\left(r_{z}\right)} .
$$

The current density of the equivalent current sheet is therefore not constant in the $w$-plane; it is modified by the scale factor, $M\left(r_{z}\right)$.

\section{Effect of Curvature on Rotor Losses}

Fig. 7 shows a quarter cross-section of a high speed PM machine, which requires a sleeve to hold the magnets, making the effective air-gap relatively large. Machine B is the same as Machine A but scaled 2:1 keeping the air-gap parameters (magnet thickness, sleeve thickness, clearance gap and slot opening) constant. The parameters of these machines are shown in Table 1. The slots of the machines shown in Fig. 7 have tooth tips, but as long as the teeth tips are not saturated, which is the case in these machines, the rectangular slot model shown in Fig. 1 remains valid (Zarko et al., 2006; Qazalbash et al., 2014a).

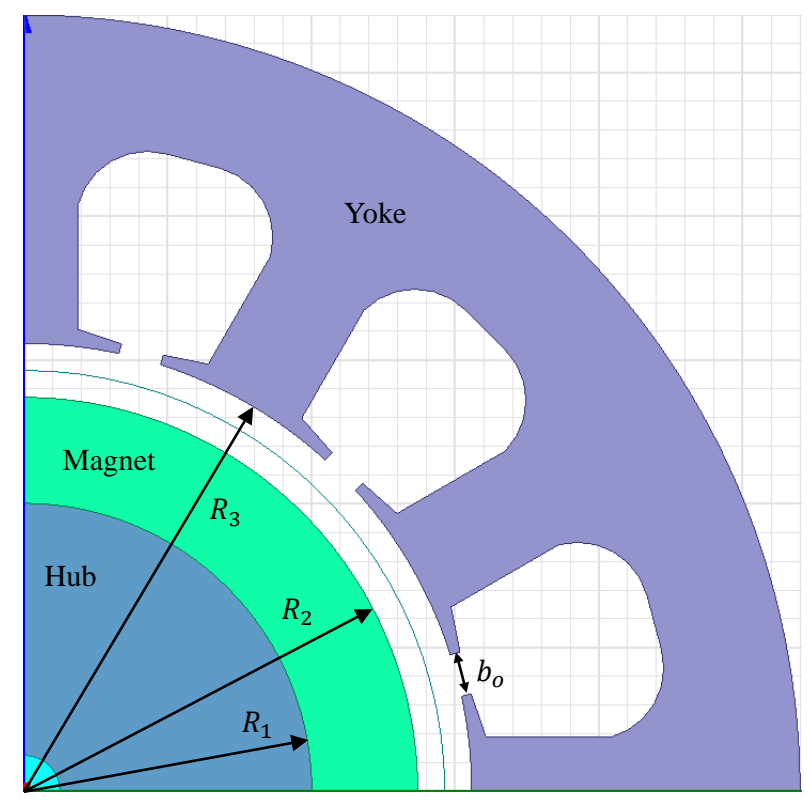

Figure 7: Quarter model of the PM synchronous machines under study.

Taking into account that the permeability of the magnets and the sleeve is close to $\mu_{0}$ the effective air-gap length as defined in Fig. 1 is:

$$
g_{z}=h_{m}+t_{s l}+h_{g}=11.12 \mathrm{~mm},
$$

where $h_{m}$ is the magnet's thickness, $t_{s l}$ the sleeve's thickness and $h_{g}$ the clearance gap as shown in Table 1 . 
Table 1: Parameters of the Machines

$\begin{array}{lccc}\text { ine Machine } & & \mathrm{A} & \mathrm{B} \\ \text { ine Rated speed } & n_{r p m} & 65 \mathrm{krpm} & 32.5 \mathrm{krpm} \\ \text { Number of poles } & 2 p & 4 & 4 \\ \text { Number of slots } & Q_{s} & 12 & 12 \\ \text { Core length } & L & 109 \mathrm{~mm} & 218 \mathrm{~mm} \\ \text { Rotor hub radius } & R_{1} & 20 \mathrm{~mm} & 47.43 \mathrm{~mm} \\ \text { Magnet outer radius } & R_{2} & 27.4 \mathrm{~mm} & 54.8 \mathrm{~mm} \\ \text { Stator radius } & R_{3} & 31.15 \mathrm{~mm} & 58.55 \mathrm{~mm} \\ \text { Magnet's thickness } & h_{m} & 7.37 \mathrm{~mm} & 7.37 \mathrm{~mm} \\ \text { Sleeve's thickness } & t_{s l} & 2.95 \mathrm{~mm} & 2.95 \mathrm{~mm} \\ \text { Clearance gap } & h_{g} & 0.8 \mathrm{~mm} & 0.8 \mathrm{~mm} \\ \text { Slot opening } & b_{o} & 3 \mathrm{~mm} & 3 \mathrm{~mm} \\ \text { Rotor hub permeability } & \mu_{r} & 750 & 750 \\ \text { Rotor hub conductivity } & \sigma_{r} & 6.7 \cdot 10^{6} \mathrm{~S} / \mathrm{m} & 6.7 \cdot 10^{6} \mathrm{~S} / \mathrm{m} \\ \text { Magnet conductivity } & \sigma_{m} & 0.77 \cdot 10^{6} \mathrm{~S} / \mathrm{m} & 0.77 \cdot 10^{6} \mathrm{~S} / \mathrm{m} \\ \text { Magnet material } & - & \mathrm{NdFeB} & \mathrm{NdFeB} \\ \text { Magnet permeability } & \mu_{r 2} & 1.07 & 1.07 \\ \text { Magnet remanence } & B_{r} & 1.05 \mathrm{~T} & 1.05 \mathrm{~T} \\ \text { Magnet coercivity } & H_{c} & 781 \mathrm{kA} / \mathrm{m} & 781 \mathrm{kA} / \mathrm{m}\end{array}$

The no-load magnetic field distribution in the air-gap of the slotless configuration in the rotor's reference frame can be expressed using complex number notation as

$$
B_{s l}(\theta, r)=\sum_{n=1,3,5}^{\infty} K_{n}(r) \cos (n p \theta)+j \sum_{n=1,3,5}^{\infty} D_{n}(r) \sin (n p \theta),
$$

where the coefficients $K_{n}(r)$ and $D_{n}(r)$ are calculated according to (Zhu et al., 2002) and $j=\sqrt{-1}$ is the imaginary unit. The complex permeance $(\mathrm{CP})$ function using the rotor's reference frame is

$$
\lambda(\theta, r, t)=\lambda_{a 0}+\sum_{m=1,2,3}^{\infty} \lambda_{a m}(r) \cos \left(m Q_{s}\left(\theta-\omega_{m} t\right)\right)+j \sum_{m=1,2,3}^{\infty} \lambda_{b m}(r) \sin \left(m Q_{s}\left(\theta-\omega_{m} t\right)\right),
$$

where the coefficients $\lambda_{a 0}, \lambda_{a m}(r)$ and $\lambda_{b m}(r)$ are calculated using conformal mapping and $\omega_{m}=\omega / p$ is the mechanical speed of the rotor. Therefore, the magnetic field distribution in the slotted geometry is

$$
B(\theta, r, t)=B_{s l}(\theta, r) \cdot \lambda^{*}(\theta, r, t)
$$

For the calculation of rotor losses we are interested in the amplitude of the asynchronous harmonics of the radial component of the magnetic field, as it discussed later in section 4.1. Therefore, combining (34), (35) and (36) we obtain:

$$
\begin{aligned}
\operatorname{Re}(B(\theta, r, t))=\sum_{n=1,3,5}^{\infty} K_{n}(r) \cos (n p \theta) & {\left[\lambda_{a 0}+\sum_{m=1,2,3}^{\infty} \lambda_{a m}(r) \cos \left(m Q_{s}\left(\theta-\omega_{m} t\right)\right)\right] } \\
& +\sum_{n=1,3,5}^{\infty} D_{n}(r) \sin (n p \theta)\left[\sum_{m=1,2,3}^{\infty} \lambda_{b m}(r) \sin \left(m Q_{s}\left(\theta-\omega_{m} t\right)\right)\right] .
\end{aligned}
$$

For a particular $m_{o}$, which means a time order $k=m_{o} Q_{s} / p$, we can re-arrange (37) to express explicitly each asynchronous harmonic. For each particular $n_{o}$ we have two asynchronous harmonics whose space orders, $q_{1}$ and $q_{2}$, 
and amplitudes, $B_{q_{1} k}$ and $B_{q_{2} k}$, are calculated as follows

$$
\begin{cases}q_{1}=k+n_{o}, & \text { and } B_{q_{1} k}=K_{n_{o}}(r) \frac{\lambda_{a m_{o}}}{2}-D_{n_{o}}(r) \frac{\lambda_{b m_{o}}}{2} \\ q_{2}=k-n_{o}, & \text { and } B_{q_{2} k}=K_{n_{o}}(r) \frac{\lambda_{a m_{o}}}{2}+D_{n_{o}}(r) \frac{\lambda_{b m_{o}}}{2}\end{cases}
$$

The two machines studied in this paper have $Q_{s}=12$ slots and $p=2$ pole pairs. Therefore, the time orders are 6 , $12,18 \ldots$

The radial and tangential components of the $\mathrm{CP}$ function on the surface of the magnets are shown in Fig. 8. In this case the value of the coefficient $K_{J}$ is

$$
K_{J}= \begin{cases}1.6 \%, & \text { for machine A } \\ 0.37 \%, & \text { for machine B, }\end{cases}
$$

which according to Fig. 3 places Machine A in the region where strong effect of the curvature is expected. Machine $\mathrm{B}$ is in the region of Fig. 3 where the effect of curvature is expected to be small. This is confirmed by Fig. 8, which shows the permeance functions of both machines with and without taking curvature into account.

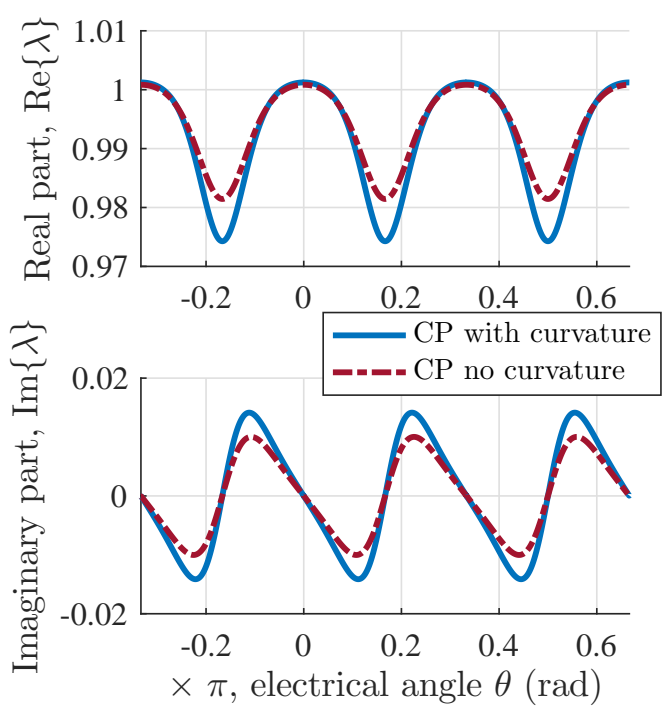

(a) Machine A.

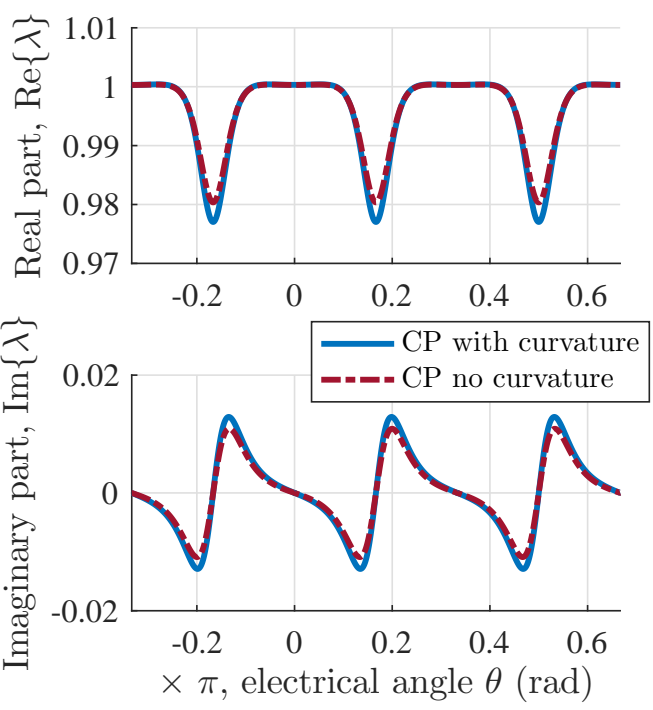

(b) Machine B.

Figure 8: Complex permeance function obtained with and without curvature; radial and tangential components.

\subsection{Calculation of No-Load Rotor Eddy-Current Power Loss}

From the perspective of the rotor's reference frame the slots change position with time and this variation of permeance produces a variation of the magnetic field seen by the rotor which induces eddy current losses, which are commonly known as tooth ripple losses (Lawrenson et al., 1966; Stoll and Sykulski, 1992). The magnitudes of the asynchronous harmonics due to tooth permeance variation can be calculated using the $\mathrm{CP}$ methods outlined earlier.

The no-load rotor eddy current power loss for each asynchronous harmonic can be calculated analytically, as described in (Sharkh et al., 2011; Qazalbash et al., 2014a,b; Zhu et al., 2004), using a cylindrical multilayer model in which each asynchronous harmonic is represented by a current sheet at the bore of a slotless stator configuration of the machine as shown in Fig. 9. The non-segmented magnet is modelled as a conducting region with no magnetization. 


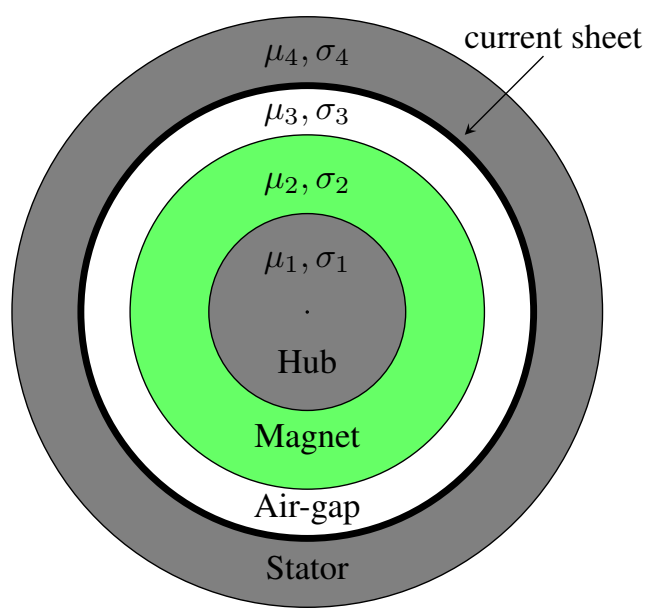

Figure 9: Cylindrical model of the PM machine with the corresponding current sheet at the stator bore.

The current sheet density that represents an asynchronous harmonic of space order $q$ and time order $k$ can be expressed as

$$
J_{q k}=\hat{J}_{q k} \cos (q \theta+k \omega t)=\operatorname{Re}\left(\hat{J}_{q k} e^{j q \theta} e^{j k \omega t}\right) .
$$

The objective is to calculate the magnetic vector potential, $A$, in the cylindrical multilayer domain shown in Fig. 9. In cylindrical coordinates the Laplacian of $A$ assuming no variation in the $z$ direction is expressed as follows:

$$
\nabla^{2} A=\frac{1}{r} \frac{\partial}{\partial r}\left(r \frac{\partial A}{\partial r}\right)+\frac{1}{r^{2}} \frac{\partial^{2} A}{\partial \theta^{2}} .
$$

Since the applied current sheet is sinusoidal in space and time, and the materials operate in their linear regions; the magnetic vector potential, $A$, can be expressed as a phasor as

$$
A(\theta, r, t)=R(r) e^{j q \theta} e^{j k \omega t},
$$

accordingly, in the steady state the Laplacian can be expressed as

$$
\nabla^{2} A=j k \omega \mu \sigma A \text {. }
$$

Substituting (42) into (43) and re-arranging the terms we obtain

$$
\frac{d^{2} R(r)}{d r^{2}}+\frac{1}{r} \frac{d R(r)}{d r}-\left(j k \omega \mu \sigma+\frac{q^{2}}{r^{2}}\right) R(r)=0,
$$

which is a modified Bessel differential equation whose general solution is given by

$$
R(\kappa r)=C I_{q}(\kappa r)+D K_{q}(\kappa r),
$$

in which $\kappa^{2}=j k \omega \mu \sigma, C$ and $D$ are constants that are determined by applying the boundary conditions, and $I_{q}$ and $K_{q}$ are the modified Bessel functions of the first and second kinds of order $q$, respectively. The radial and tangential components of the magnetic field distribution are calculated as follows:

$$
\begin{aligned}
& B_{r}(\theta, r, t)=\frac{1}{r} \frac{\partial A}{\partial \theta}=\frac{1}{r} j q\left[C I_{q}(\kappa r)+D K_{q}(\kappa r)\right] e^{j q \theta} e^{j k \omega t}, \\
& B_{\theta}(\theta, r, t)=-\frac{\partial A}{\partial r}=-\kappa\left[C I_{q}^{\prime}(\kappa r)+D K_{q}^{\prime}(\kappa r)\right] e^{j q \theta} e^{j k \omega t} .
\end{aligned}
$$

The constants $C$ and $D$ for each layer (1 rotor hub, 2 magnets, 3 air-gap and 4 stator iron) are obtained after applying the following boundary conditions: 
1. $B_{r}$ and $B_{\theta}$ are finite as $r$ approaches zero,

2. $B_{r}$ is zero at the outer radius of the stator,

3. the radial flux density, $B_{r}$, is continuous at all interfaces,

4. the tangential field intensity, $H_{\theta}$, is continuous at $r=R_{1}$ and $r=R_{2}$,

5. at the current sheet $\left(r=R_{3}\right.$ ) there is a discontinuity in the tangential field intensity, $H_{\theta}$, by the amount of the current sheet.

Once the constants are calculated the magnetic vector potential is known in all the domain and the currents induced in the axial direction are calculated as follows:

$$
J_{\text {axial }}=-\sigma \frac{\partial A}{\partial t},
$$

where $\sigma$ is the conductivity of the material. The total rotor losses can be obtained by integrating $J_{\text {axial }}^{2} / \sigma$ in all the domain or using the Poynting vector (Qazalbash et al., 2014a,b; Zhu et al., 2004).

The amplitude of the current sheet that corresponds to each asynchronous harmonic, $\hat{J}_{q k}$, is effectively set to produce the same normal flux density on the surface of the magnet $\hat{B}_{q k}$ (Qazalbash et al., 2014a,b, 2015). In practice, the problem is solved by setting $\hat{J}_{q k}=1$ and calculating the corresponding losses $P_{q k 1}$ from the solution of the diffusion equation in the current sheet model. In addition, the Laplace equation (no eddy currents) is solved to find the corresponding $\hat{B}_{q k 1}$ when $\hat{J}_{q k}=1$. Finally, the actual losses for a given $\hat{B}_{q k}$ (obtained from harmonic analysis using conformal mapping in this case) are calculated as

$$
P_{q k}=\left(\frac{\hat{B}_{q k}}{\hat{B}_{q k 1}}\right)^{2} P_{q k 1}
$$

\subsubsection{Limitations of the complex permeance and current sheet model}

The current sheet model provides more than reasonable results for the calculation of eddy currents as long as the amplitudes of the asynchronous harmonics are accurate. However, there are several simplifying assumptions.

First, the current sheet model is two-dimensional. Therefore, the end effects and the influence of the return path of the eddy currents induced is neglected. The model does not cater for peripheral magnet segmentation.

In machines with relatively small active length to diameter ratios, it is necessary to use 3D FEA because the end effects can be significant (Hendershot and Miller, 2010; Russell and Norsworthy, 1958). Nevertheless, the qualitative information and the insight provided by the current sheet model in these case can be helpful to a machine designer.

The properties of the materials are assumed to be linear and isotropic. Accordingly, the methodology presented in this paper does not take into account the saturation. Significant saturation, particularly of the tooth tip can result in significant under estimation of the magnitudes of the asynchronous harmonics and corresponding losses (Qazalbash et al., 2014a,b, 2015).

Finally, the effect of the induced eddy currents on the traveling flux harmonics is neglected, the eddy currents generate travelling harmonics that interact with the slotting, which is not taken into account in the slotless current sheet model. However, this effect is expected to be negligible due to the large air-gap and the relatively weak magnetic field of the eddy currents (Irenji, 1998; Qazalbash, 2014).

\subsection{Transient FEA}

Transient FEA was used to calculate rotor losses directly. A constant mechanical speed was assigned to the rotor without any other excitation besides the PM magnetisation. In this case, the element size was determined such that there are at least 3 elements per skin depth. The model had around 100 thousand elements. for accurate calculation of eddy currents. The time step was set to $0.5 \mu$ s such that there are at least 10 time steps per slot opening so that 
the slotting permeance variation is captured at a high resolution. Mesh size and time step independence were also confirmed.

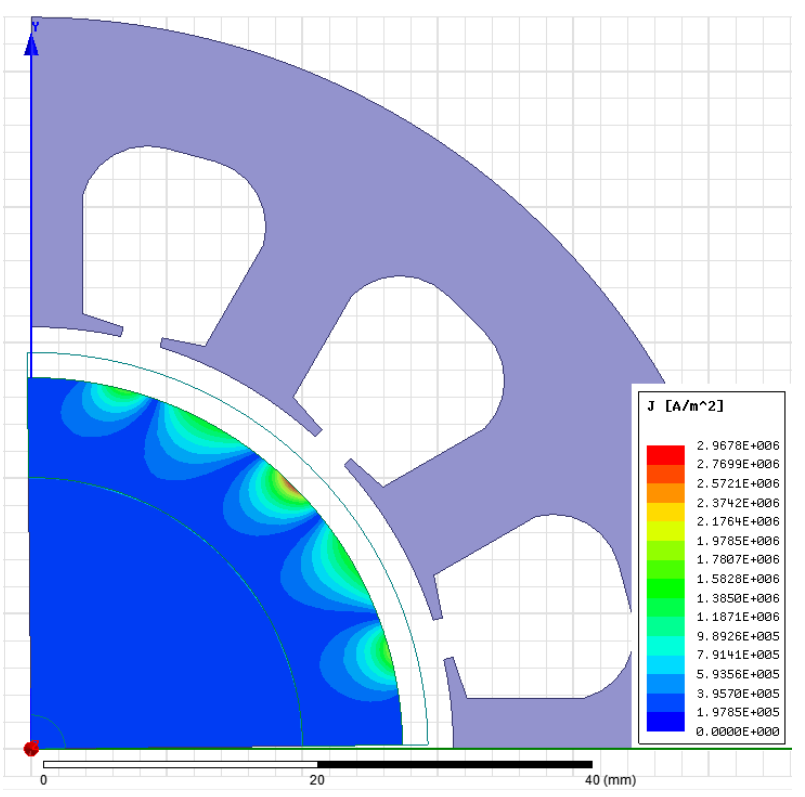

Figure 10: Eddy current density at a particular instant of time obtained using transient FEA, Machine A shown as an example.

Fig. 10 shows the eddy current density obtained from the transient FEA simulation. It can be appreciated that most of the rotor losses are concentrated in the magnets.

\subsection{Results and Discussion}

Fig. 11 shows the waveform of the radial component of the no-load magnetic field distribution on the surface of the magnet at a particular rotor position using two-dimensional static FEA, CP function with curvature and CP function without curvature. It can be appreciated in Fig. 11 that in machine $\mathrm{A}$ the $\mathrm{CP}$ function without curvature underestimates the amplitude of the tooth-ripple harmonics. On the other hand, in the case of machine B; which has smaller $K_{J}$, this effect is less significant.

Ignoring the effect of curvature underestimates the amplitude of the asynchronous harmonics in both cases as shown in Fig. 12. However, in Machine A this effect is more significant.

The no-load rotor losses obtained using transient FEA are shown in Fig. 13. Table 2 shows a comparison of the average no-load rotor losses obtained with the linear transient FEA, the CP function taking into account the effect of curvature and the $\mathrm{CP}$ function neglecting the effect of curvature for the machines under study running at rated speed. The no-load rotor losses were calculated using the current sheet model described in section 4.1.

Table 2: No-load rotor power loss

$\begin{array}{lccc}\text { ine Machine } & & \text { A } & \text { B } \\ \text { ine Transient FEA } & \approx & 25.4 \mathrm{~W} & 76.2 \mathrm{~W} \\ \text { Model with curvature } & \approx & 22.7 \mathrm{~W} & 87.0 \mathrm{~W} \\ \text { Model without curvature } & \approx & 12.7 \mathrm{~W} & 67.0 \mathrm{~W} \\ \text { ine } & & & \end{array}$

The no-load rotor losses obtained using the $\mathrm{CP}$ function without curvature are significantly lower than the result obtained using FEA. On the other hand, the value obtained when the effect of curvature is taken into account agrees 


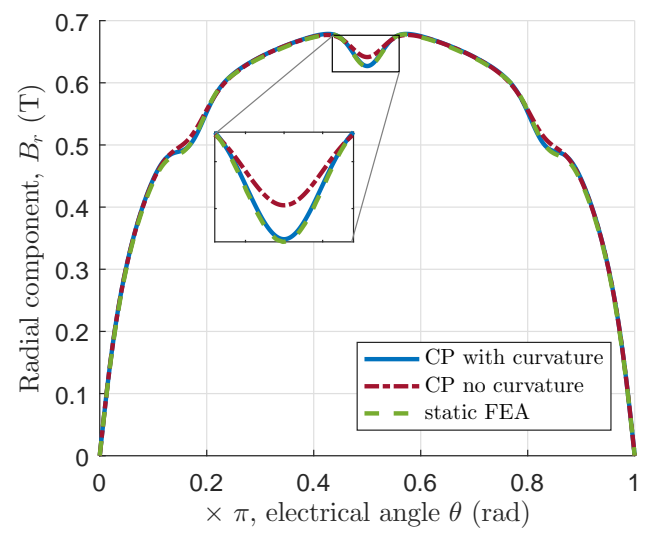

(a) Machine A.

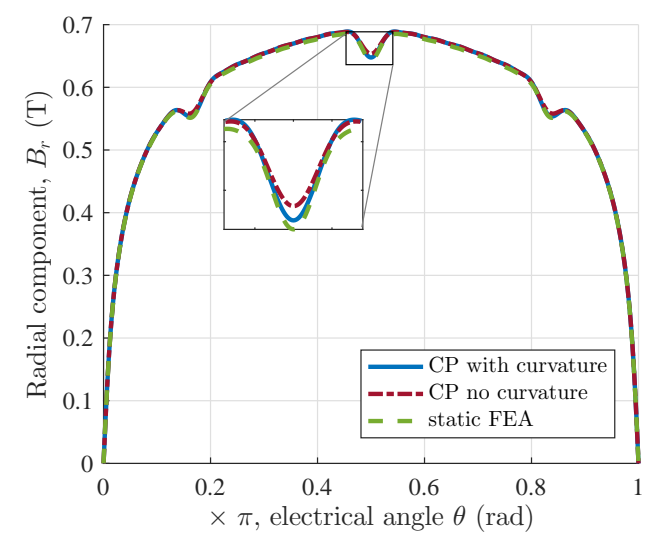

(b) Machine B.

Figure 11: Magnetic field distribution in the air-gap obtained using two-dimensional static FEA and the CP function with and without curvature.

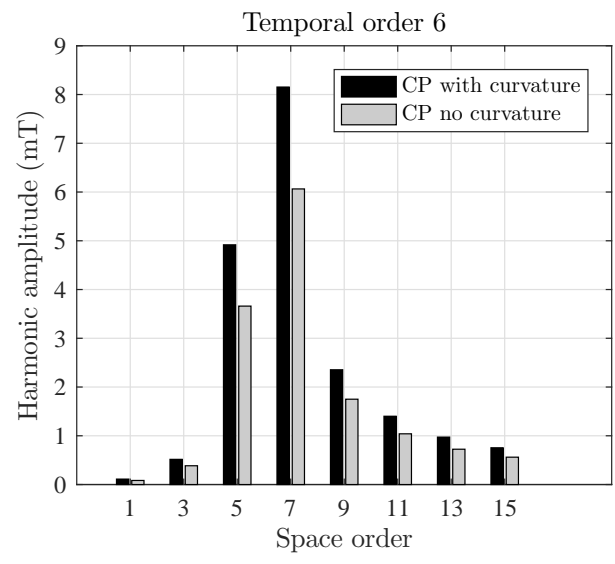

(a) Machine A.

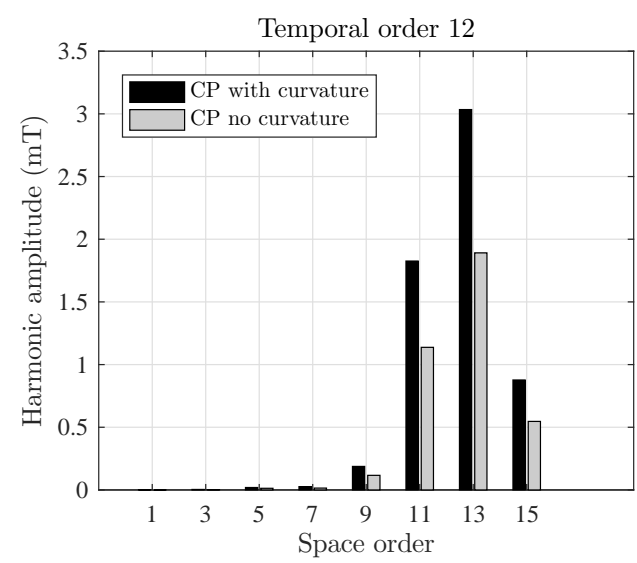

(c) Machine A.

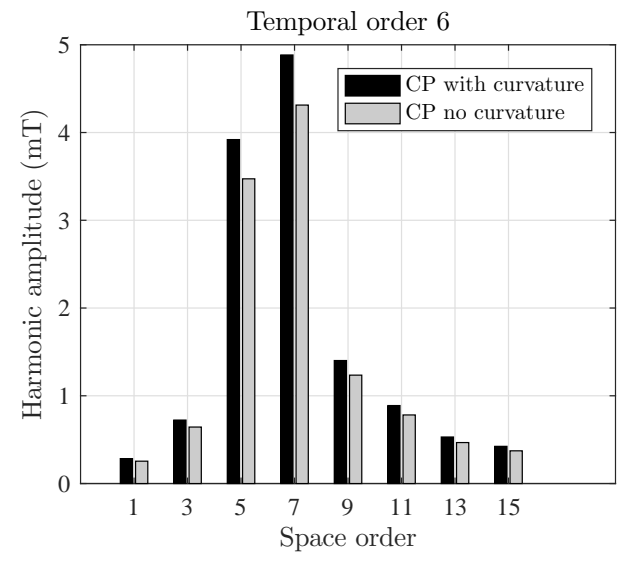

(b) Machine B.

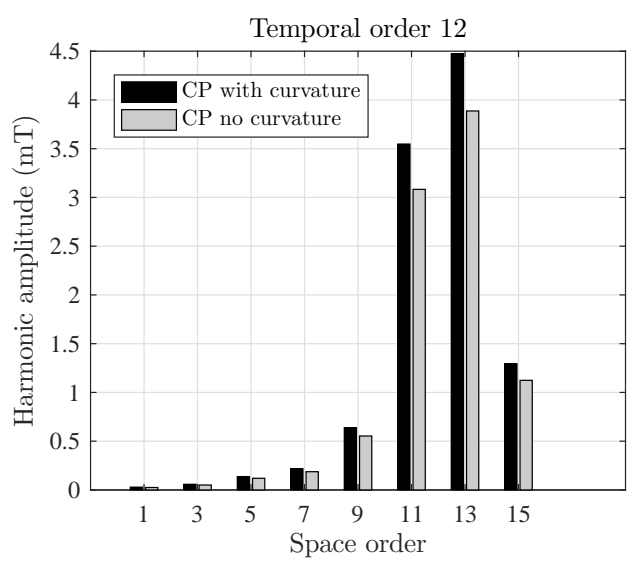

(d) Machine B.

Figure 12: Amplitudes of significant magnetic induction space harmonics, Machine A is running at $65000 \mathrm{rpm}$ and Machine B is running at $32500 \mathrm{rpm}$. 


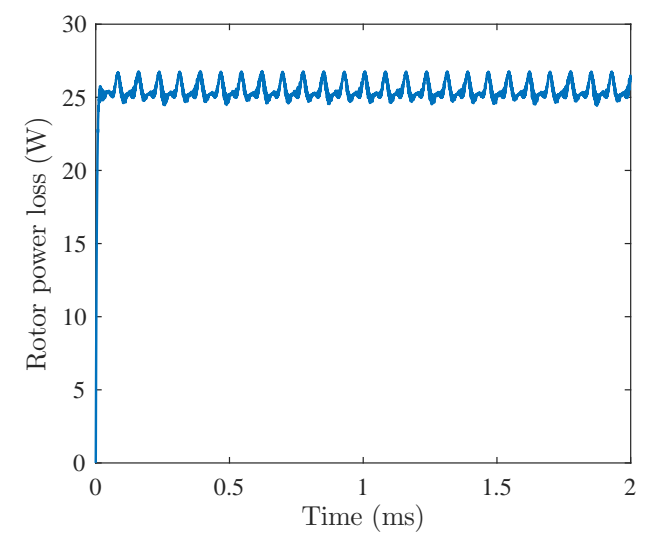

(a) Machine A.

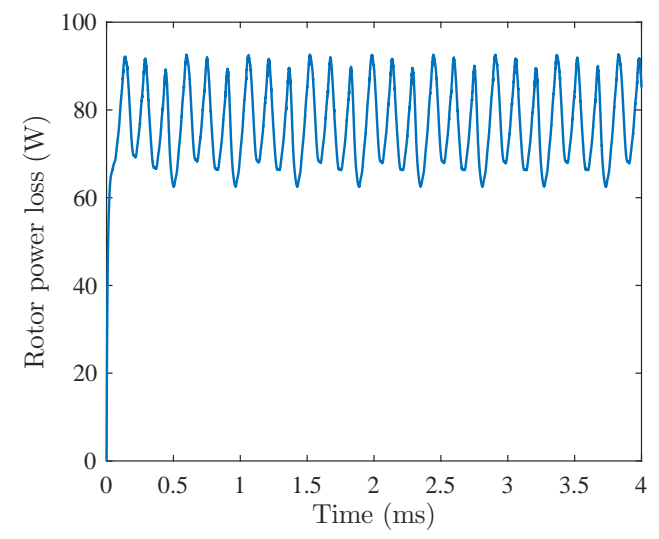

(b) Machine B.

Figure 13: No-load rotor power loss at rated speeds calculated using transient FEA.

reasonably well with FEA. In Machine A, the model with curvature underestimates the losses by a factor of 0.89 compared to the model without curvature, which underestimates losses by a factor of 0.5 . In machine $\mathrm{B}$, the model with curvature overestimates the losses by a factor of 1.14 and in the model without curvature underestimates the losses by a factor of 0.88 . This is consistent with the fact that the curvature coefficient is higher in Machine A than in Machine B and as expected the discrepancy between the results neglecting curvature and FEA is greater in Machine A.

The difference between FEA and the model with curvature may be explained to be due to numerical errors as well as the assumptions made in the current sheet model as discussed in section 4.1.1.

\section{Conclusion}

In this paper the influence of curvature on the magnetic field distribution and no-load rotor losses in slotted rotating PM electric machines has been investigated. The proposed proportional logarithmic transformation provides an insight into the effect of curvature because it preserves the length scale. The curvature coefficient, $K_{J}$, indicates when the effect of curvature is going to be important.

The value of the curvature coefficient, $K_{J}$ which is the ratio of the air-gaps in the rectangular and cylindrical models in the $w$ and $z$-planes, respectively, tends to be small. However, this small change of the air-gap length in the $w$-plane has a significant influence in the amplitude of the asynchronous harmonics of the magnetic field distribution as shown in Fig. 12.

The case study presented in Section 4 illustrates how strong the effect of curvature can be in machines with a large effective air-gap, particularly on rotor losses. Ignoring the effect of curvature grossly underestimates the no-load eddy current losses in the rotor in Machine A with large ratio of air-gap length to radius ratio. On the other hand, the effect of curvature is less significant in Machine B as anticipated from the smaller value of $K_{J}$.

\section{References}

Boughrara, K., Chikouche, B. L., Ibtiouen, R., Zarko, D., and Touhami, O. (2009). Analytical model of slotted airgap surface mounted permanent-magnet synchronous motor with magnet bars magnetized in the shifting direction. IEEE Transactions on Magnetics, 45(2):747-758. 
Boules, N. (1985). Prediction of no-load flux density distribution in permanent magnet machines. IEEE Transactions on Industry Applications, IA-21(3):633-643.

Carter, F. (1900). Note on air-gap and interpolar induction. Journal of the Institution of Electrical Engineers, 29(146):925-933.

Carter, F. (1926). The magnetic field of the dynamo-electric machine. Journal of the Institution of Electrical Engineers, 64(359):1115-1138.

Dubas, F. and Espanet, C. (2009). Analytical solution of the magnetic field in permanent-magnet motors taking into account slotting effect: No-load vector potential and flux density calculation. IEEE Transactions on Magnetics, 45(5):2097-2109.

Fitzgerald, A. E., Kingsley, C., and Umans, S. D. (2003). Electric Machinery. McGraw-Hill.

Freeman, E. M. (1962). The calculation of harmonics, due to slotting, in the flux-density waveform of a dynamoelectric machine. Proceedings of the IEE - Part C: Monographs, 109(16):581-588.

Gibbs, W. J. (1958). Conformal Transformations in Electrical Engineering. Chapman and Hall.

Hammond, P. (1999). The role of the potentials in electromagnetism. COMPEL - The international journal for computation and mathematics in electrical and electronic engineering, 18(2):103-119.

Hendershot, J. R. and Miller, T. J. E. (2010). Design of Brushless Permanent-Magnet Machines. Motor Design Books LLC.

Irenji, N. T. (1998). Calculation of Electromagnetic Rotor Losses in High-Speed Permanent Magnet Machines. PhD Thesis, University of Southampton.

Krause, P., Wasynczuk, O., Sudhoff, S., and Pekarek, S. (2013). Analysis of Electric Machinery and Drive Systems, Appendix B: Carter's Coefficient. John Wiley \& Sons, Inc.

Kundur, P. (1993). Power System Stability and Control. McGraw-Hill.

Lawrenson, P. J., Reece, P., and Ralph, M. C. (1966). Tooth-ripple losses in solid poles. Proceedings of the Institution of Electrical Engineers, 113(4):657-662.

Markovic, M., Jufer, M., and Perriard, Y. (2004). Analyzing an Electromechanical Actuator by Schwarz-Christoffel Mapping. IEEE Transactions on Magnetics, 40(4):1858-1863.

Markovic, M., Jufer, M., and Perriard, Y. (2005). An exact formula for "Tooth-to-Tooth" permeance. IEEE Transactions on Magnetics, 41(7):2295-2299.

Matsch, L. W. (1972). Electromagnetic and Electromechanical Machines. Intext Educational Publishers.

Qazalbash, A. A. (2014). Rotor eddy current power losses in high speed permanent magnet synchronous generators. $\mathrm{PhD}$ Thesis, University of Southampton.

Qazalbash, A. A., Sharkh, S. M., Irenji, N. T., Wills, R. G., and Abusara, M. A. (2014a). Calculation of no-load rotor eddy-current power loss in pm synchronous machines. IEEE Transactions on Magnetics, 50(9):1-8.

Qazalbash, A. A., Sharkh, S. M., Irenji, N. T., Wills, R. G., and Abusara, M. A. (2014b). Rotor eddy current power loss in permanent magnet synchronous generators feeding uncontrolled rectifier loads. IEEE Transactions on Magnetics, 50(6):1-9.

Qazalbash, A. A., Sharkh, S. M., Irenji, N. T., Wills, R. G., and Abusara, M. A. (2015). Rotor eddy loss in high-speed permanent magnet synchronous generators. IET Electric Power Applications, 9(5):370-376.

Rabinovici, R. (1996). Magnetic field analysis of permanent magnet motors. IEEE Transactions on Magnetics, 32(1):265-269. 
Russell, R. L. and Norsworthy, K. H. (1958). Eddy currents and wall losses in screened-rotor induction motors. Proceedings of the IEE - Part A: Power Engineering, 105(20):163-175.

Sharkh, S. M., Qazalbash, A. A., Irenji, N. T., and Wills, R. G. (2011). Effect of slot configuration and airgap and magnet thicknesses on rotor electromagnetic loss in surface pm synchronous machines. In International Conference on Electrical Machines and Systems (ICEMS), 2011, pages 1-6.

Stoll, R. and Sykulski, J. (1992). Modelling tooth ripple losses in the solid pole faces of synchronous machines. COMPEL - The international journal for computation and mathematics in electrical and electronic engineering, 11(1):105-108.

Wu, L. J., Zhu, Z. Q., Staton, D., Popescu, M., and Hawkins, D. (2010). Comparison of analytical models for predicting electromagnetic performance in surface-mounted permanent magnet machines. In 2010 IEEE Vehicle Power and Propulsion Conference, pages 1-6.

Wu, L. J., Zhu, Z. Q., Staton, D., Popescu, M., and Hawkins, D. (2011). An improved subdomain model for predicting magnetic field of surface-mounted permanent magnet machines accounting for tooth-tips. IEEE Transactions on Magnetics, 47(6):1693-1704.

Wu, L. J., Zhu, Z. Q., Staton, D., Popescu, M., and Hawkins, D. (2012). Analytical model of eddy current loss in windings of permanent-magnet machines accounting for load. IEEE Transactions on Magnetics, 48(7):2138-2151.

Zarko, D., Ban, D., and Lipo, T. A. (2006). Analytical calculation of magnetic field distribution in the slotted air gap of a surface permanent-magnet motor using complex relative air-gap permeance. IEEE Transactions on Magnetics, 42(7):1828-1837.

Zhu, Z., Wu, L., and Xia, Z. (2010). An Accurate Subdomain Model for Magnetic Field Computation in Slotted Surface-Mounted Permanent-Magnet Machines. IEEE Transactions on Magnetics, 46(4):1100-1115.

Zhu, Z. Q. and Howe, D. (1993). Instantaneous magnetic field distribution in brushless permanent magnet dc motors. iii. effect of stator slotting. IEEE Transactions on Magnetics, 29(1):143-151.

Zhu, Z. Q., Howe, D., and Chan, C. C. (2002). Improved analytical model for predicting the magnetic field distribution in brushless permanent-magnet machines. IEEE Transactions on Magnetics, 38(1):229-238.

Zhu, Z. Q., Ng, K., Schofield, N., and Howe, D. (2004). Improved analytical modelling of rotor eddy current loss in brushless machines equipped with surface-mounted permanent magnets. IEE Proceedings - Electric Power Applications, 151(6):641-650. 